Ruiz, L., Camargo, D. y Cardona, O. (2020), Cambios en el valor bursátil de empresas colombianas listadas: un análisis del antes y el después de la convergencia a NIIF. Contaduría Universidad de Antioquia, 76, 97-114.

Doi: https://doi.org/10.17533/udea.rc.n76a04

\title{
Cambios en el valor bursátil de empresas colombianas listadas: un análisis del antes y el después de la convergencia a NIIF*
}

\author{
Liliana Elizabeth Ruiz Acosta \\ liliana.ruiz@unimilitar.edu.co \\ Universidad Militar Nueva Granada \\ orcid: 0000-0003-3323-8480 \\ David Andrés Camargo Mayorga \\ david.camargo@unimilitar.edu.co \\ Universidad Militar Nueva Granada \\ orcid: 0000-0002-5290-8251 \\ Octavio Cardona García \\ octavio.cardona.g@gmail.com \\ Universidad Militar Nueva Granada \\ orcid: 0000-0001-6257-5270
}

\footnotetext{
Artículo de investigación derivado del proyecto INV-ECO 2330 "Análisis de las prácticas organizacionales producidas en el proceso de convergencia a normas internacionales de información financiera en el Banco Colpatria-Multibanca Colpatria" financiado por la Vicerrectoría de Investigaciones de la Universidad Militar Nueva Granada en vigencia 2017.
} 
Cambios en el valor bursátil de empresas colombianas listadas: un análisis del antes y el después de la convergencia a NIIF

Resumen: Este artículo tiene como objetivo examinar el efecto que tuvo la convergencia a Normas Internacionales de Información Financiera (NIIF) sobre el valor bursátil de 50 empresas que cotizan en la Bolsa de Valores de Colombia (BVC). Las empresas en Colombia hicieron tránsito desde los principios de contabilidad generalmente aceptados en Colombia - GAAP (Decreto 2649 de 1993) hacia las NIIF (Ley 1314 de 2009), que con sus decretos reglamentarios acogieron las Normas Internacionales de Información Financiera (NIIF) de forma obligatoria desde el año 2014. Este estudio es de corte cuantitativo y compara mediante modelos de regresión lineal la información de los Estados Financieros de 50 empresas para dos años antes (2013 y 2014) y dos después (2015 y 2016) de la convergencia a NIIF. Se observa que no existe una fuerte relación entre el valor bursátil de las acciones de las empresas y la información presentada en sus Estados Financieros.

Palabras clave: Valor Bursátil, NIIF, Economías Emergentes, Mercado de Capitales.

Changes in stock value of listed Colombian companies: an analysis of their situation before and after convergence to IFRS

Abstract: This paper aims at examining the effect convergence to the International Financial Reporting Standards (IFRS) had on the stock value of 50 companies listed on the Colombia Stock Exchange (BVC). Colombian companies made a transition from the Generally Accepted Accounting Principles - GAAP (Decree 2649 of 1993) to IFRS (Law 1314 of 2009), which adopted the International Financial Reporting Standards (IFRS) through their regulatory decrees, in a mandatory way from the year 2014. This is a quantitative study which compares the information on the Financial Statements of 50 companies for two years before (2013 and 2014) and two years after (2015 and 2016) convergence to the IFRS by means of linear regression models. It is observed that there is not a strong relationship between the stock value of the company shares and the information presented in their Financial Statements.

Keywords: Stock value, IFRS, Emerging economies, Capital market.

Mudanças no valor acionário de empresas colombianas listadas: uma análise do antes e o depois da convergência a NIIF

Resumo: Este artigo tem como objetivo examinar o efeito que teve a convergência a Normas Internacionais de Informação Financeira (NIIF) sobre o valor acionário de 50 empresas que tem cotação na Bolsa de Valores da Colômbia (BVC). As empresas na Colômbia fizeram um trânsito desde os princípios de contabilidade geralmente aceites na Colômbia - FAAp (Decreto 2649 de 1993) para as NIIF (Lei 1314 de 2009), que com os seus decretos regulamentares acolheram as Normas Internacionais de Inforamação financeira (NIIF) de forma obrigatória desde o ano 2014. Este estudo é do tipo quantitativo e compara a através de modelos de regressão linear a informação dos Estados Financeiros de 50 empresas para dois anos antes (2013 e 2014) e dois depois (2015 e 2016) da convergência a NIIF. Observa-se que não existe uma relação forte entre o valor acionário das empresas na Bolsa de Valores e a informação apresentada nos seus Estados Financeiros.

Palavras-Chave: Valor acionário, NIIF, Economias Emergentes, Mercado de capitais

Des changements dans la valeur boursière des entreprises colombiennes en liste : une analyse de l'avant et de l'après de la convergence aux IFRS

Résumé: le but de cet article est d'examiner l'effet de la convergence aux Normes Internationales d'Information Financière (IFRS) sur la valeur bursière de 50 entreprises qui cotent dans la Bourse de Valeur Colombienne (BVC). Les entreprises en Colombie ont fait transit depuis les principes de comptabilité accepté généralement en Colombie- GAAP (Decret 2649 de 1993) vers les IFRS (Loi 1314 de 2009). Avec leurs decrét réglementaires, elles ont adopté les Normes Internationales d'Information Financière (IFRS) de manière obligatoire depuis l'an 2014. Cette étude est d'une approche quantitative; elle compare au moyen des modèles de régression linéaire l'information des États Financiers de 50 entreprises de deux ans avant (2013 et 2014) et deux ans après (2015 et 2016) s'être converties aux IFRS. Il a été observé qu'il n'existe pas un rapport fort entre la valeur boursière des actions des entreprises et l'information présentée dans leurs État Financiers.

Mots clés: valeur boursière, IFRS, économies émergentes, marchés des capitaux 
Cont. udea (enero-junio), pp. 97-114. (C) Universidad de Antioquia-2020.

\title{
Cambios en el valor bursátil de empresas colombianas listadas: un análisis del antes y el después de la convergencia a NIIF
}

\author{
Liliana Elizabeth Ruiz Acosta, David Andrés Camargo Mayorga, \\ Octavio Cardona García \\ https://doi.org/10.17533/udea.rc.n76a04
}

Primera versión recibida en enero de 2020 - Versión aceptada en marzo de 2020

\section{Introducción}

L

a información financiera es uno de los mensajes contables con mayor difusión e influencia en el mundo de los negocios; se adapta y ajusta a cambios normativos y culturales, y recientemente la estandarización hacia Normas Internacionales de Información Financiera (NIIF) la ha modificado. Para Jarne (1997) la información contable es el medio de transmisión de la realidad empresarial, que surgió para satisfacer a los usuarios en lo relativo a la comprensión y análisis de la información proveniente de cada empresa, y que es determinante en la actualidad para el proceso de integración de las economías a nivel mundial.

Mhedhbi y Zeghal (2016) consideran que la contabilidad internacional nació como una estrategia que genera información financiera de alta importancia y utilidad a nivel global, lo que según Rueda (2013), se desarrolló con el propósito de conducir la globalización y la internacionalización por el mismo camino, generando una estabilidad financiera que impulsa la inversión y la libre circulación del capital. Además, para que fuera una herramienta para la reducción de riesgos de inversión, aumentando la transparencia y la confianza por los mercados de capitales (Zaidi y Paz, 2015).

En la misma línea argumental, El-Sayed (2016), plantea que la contabilidad internacional surgió con el paso de los negocios de la esfera local a la global, donde los interesados: empresas, inversionistas, reguladores, accionistas, prestamistas, proveedores, gerentes, sindicatos y empleados, entre otros, evidenciaron la necesidad de generar un conjunto de normas comunes, 
Ruiz, L., Camargo, D. y Cardona O. Cambios en el valor bursátil de empresas colombianas listadas...

mediante las cuales se diera a conocer la información de las compañías bajo unos estándares similares. De acuerdo con Chandler y Holzer (1984), dentro de un sistema contable interactúan además de las empresas, otros actores que demandan cada vez más información financiera (Ball, 2016) para la toma de decisiones.

Para Weirich, Avery y Anderson (1971), la contabilidad internacional tiene como objetivo mejorar las prácticas contables para conformar un sistema adaptable a todos los países del mundo, bajo los mismos principios y un mismo lenguaje. Según Nobes y Parker (2006), esta rama de la contabilidad se encuentra encaminada a generar un análisis comparativo que supla las necesidades de información contable en los mercados financieros internacionales, debido a que los inversionistas, nacionales o extranjeros, la necesitan conocer en términos más entendibles (Mhedhbi y Zeghal, 2016).

Para Callao y Jarne (1995), lo relevante de la información contable en el contexto internacional está en ser un medio de comunicación entre la empresa y los usuarios que toman decisiones. Sin embargo, para Gómez (2004), el proceso de regulación contable internacional se ha centrado en los mercados financieros, desdibujando la función de control que tiene la contabilidad financiera.

En cualquier caso, la contabilidad internacional "(...) surge como una consecuencia de la internacionalización de la actividad económica" (Rodríguez, 2003, p. 1), al mismo tiempo que es producida por la dificultad para decidir con base en información y lenguajes revelados por las empresas de manera heterogénea, y por los retrasos en los informes financieros y de alta confiabilidad que propicien la atracción de inversores foráneos. Los agentes económicos deciden a partir de información producida con prácticas contables aceptadas en los países desarrollados (Halbouni, 2005), bajo lógicas que para Hamidah et al. (2015), obedecen a intereses hegemónicos internacionales, porque según Lasmin (2012), los sistemas de contabilidad originarios de los países en vía de desarrollo tienen debilidades manifiestas que dificultan la toma de decisiones de inversión.

Frente a esto, Ball (2006), manifiesta de forma crítica que los entornos institucionales y la mejora en ellos pueden hacer mejor la calidad de la información financiera que el apoyarse en un conjunto de normas contables de aceptación internacional.

Para Carnegie y Napier (2002), a la contabilidad vista como una actividad social e institucional, al convertirse en internacional, se le adjudica el objetivo de armonizar las prácticas contables nacionales con las mundialmente aceptadas, mediante comparativos que según Halbouni (2005) persiguen información cada vez más detallada y precisa acerca del desempeño y potencialidad de cada empresa. 
En síntesis, con la adopción de la contabilidad internacional se favorece a los inversores, principalmente de tres maneras: la primera, con una reducción de los costos de generación y transmisión de la información contable, por la existencia de un solo marco que hace que los Estados Financieros sean comparables (Barth et al. 2012; Yip y Young, 2012); la segunda, con una reducción de incertidumbre por la adhesión a estándares claros y precisos (Zaidi y Paz, 2015); y la tercera, porque se dota a los inversionistas de una herramienta objetiva, no sesgada, ni comprometida con ideologías (Rueda, 2013).

En últimas, como lo sostiene El-Sayed (2016), la contabilidad internacional influye directamente en los mercados de capitales, ya que los inversionistas se basan en la evaluación del desempeño de la organización y los Estados Financieros para sus análisis y decisiones de inversión bursátil e, incluso, como lo señala Botzem (2014), incide en la actividad empresarial y en el emprendimiento transnacional.

Dentro de lo que se investiga en contabilidad internacional, está la línea de trabajo en armonización que, combinada con el estudio de los estándares, que son del dominio de la contabilidad financiera, dan lugar a las investigaciones sobre la armonización internacional de normas de contabilidad financiera (Baker y Barbu, 2007); es decir, la implementación de las NIIF (o IFRS por sus siglas en inglés).

Países como Canadá y Sudáfrica tomaron la decisión de adoptar las NIIF, tal y como el Consejo de Estándares Internacionales de Contabilidad (IASB por sus siglas en inglés) hizo su recomendación de uso y en los tiempos brindados por esta entidad, mientras que la Unión Europea las introdujo mediante un proceso híbrido de aceptación de parte de las normas nacionales, que dio como resultado el uso de estándares diferentes a los promovidos por el IASB; en el caso de Japón la adopción se dio con plazo promedio de 7 años y con algunos ajustes, lo cual supuso una adopción heterogénea de las NIIF (Begoña, 2014). Esas diferencias son la consecuencia de entornos políticos y sociales disímiles, que inciden sobre los procesos reguladores y las prácticas contables de cada país (Callao y Jarne, 1995).

Por ende, se entiende el proceso de convergencia contable como un paso anclado a la globalización económica y financiera, que suministra las herramientas necesarias para la libre circulación de capital, convirtiéndose en el medio para eliminar las diferencias en el reporte de la información financiera para la expansión del capital, así como un instrumento de política económica que hace más propicia la internacionalización de una economía (Banco Mundial, 2005).

En el caso de Colombia, la Ley 1314 de 2009 planteó la convergencia a estándares de aceptación mundial. Se pasó del Decreto 2649 de 1993, que establecía principios de contabilidad generalmente aceptados PCGA, a las NIIF. 
Ruiz, L., Camargo, D. y Cardona O. Cambios en el valor bursátil de empresas colombianas listadas...

Considerando que uno de los principales objetivos de la Ley 1314 de 2009 consistía en mejorar el desarrollo de la actividad empresarial, al tiempo que buscaba que los informes contables fueran más comprensibles y homogéneos para los usuarios de la información que así lo requerían, este artículo examina la relación existente entre la información financiera y el valor bursátil de las acciones de 50 empresas listadas en la BVC dos años antes y dos después de las NIIF, toda vez que la acción cotizada, constituye el indicador del valor de una compañía en el mercado de capitales. En ese sentido, observar si la información contable afecta el valor de la acción y constituye un elemento fundamental para determinar el efecto de la aplicación de NIIF en el país.

Este documento se ubica dentro de la literatura existente sobre la adopción de las NIIF, en el apartado de efectos en el mercado de capitales según la taxonomía trabajada por Houqe (2018).

Para los fines propuestos este documento se divide en cinco secciones: la primera hace una introducción al tema; la segunda, una revisión de antecedentes; la tercera presenta la metodología; la cuarta exhibe los resultados obtenidos; y la quinta enseña las conclusiones.

\section{Antecedentes}

En la literatura, la investigación sobre los efectos de la adopción de las NIIF es diversa. De acuerdo con De George, Li y Shivakumar (2016), en una primera etapa de la producción científica en este tema, las NIIF ofrecían beneficios para las empresas y los países que las adoptaban según las circunstancias, porque había mayor transparencia de la información financiera, menores costos de capital, más inversiones foráneas, y se podían comparar los reportes contables con más facilidad. Aspectos en los que coincide Houqe (2018), que son atribuibles a la reducción de las asimetrías de información y la comparabilidad.

Sin embargo, para De George, Li y Shivakumar (2016), estudios ulteriores le atribuyen parte de los beneficios de las NIIF, a factores distintos que tienen que ver con las múltiples formas de aplicación de los estándares y no a las NIIF per se.

Houqe (2018) identificó once temas en total en los que se agrupa la producción científica en este tema. Estos son: la comparabilidad normativa contable entre países; los efectos sobre comercio exterior y las inversiones; la incidencia sobre el valor revelado de las empresas; la gestión de las ganancias a partir de los reportes financieros y la estructuración de transacciones; la cautela contable al informar ganancias y activos; los pronósticos de los analistas contables; y los efectos en el mercado de capitales sobre la liquidez de mercado, el costo de capital, el costo de la deuda y en el desempeño de la compañía.

De lo anterior, se observa que existen opiniones divididas respecto a los impactos de la contabilidad internacional, y también sobre la complejidad que envuelve la implementación de las NIIF. La cuestión no es de convicción para 
adoptar estos estándares, sino más bien de aceptación a la sumisión; lo que deben asumir quienes deciden hacerlo, enfrentando los retos que esto implica (Botzem, 2014).

Autores como Ball (2016) sostienen que los efectos al finalizar la adopción de NIIF tienden a ser relativamente insignificantes y difíciles de detectar, pero otras posturas señalan que los beneficios que trae la implementación y aceptación de normas o procedimientos contenidos en la contabilidad internacional, mejoran variables como los ingresos percibidos por las compañías, porque en la contabilidad internacional se reflejan las ganancias en términos de relevancia, lo que mueve a los inversionistas a realizar transacciones e invertir sus recursos.

Tal y como lo indican Mhedhbi y Zeghal (2016, p. 257), "La utilización de normas contables de alta calidad y altamente recomendables constituye un factor importante para hacer los informes de contabilidad más creíbles", por tanto, se facilita la divulgación de información sobre el desempeño organizacional bajo parámetros mundialmente aceptados, porque la información contable tiende a ser más completa y específica que la producida con normas locales (El-Sayed, 2016).

Rueda (2010) afirma que además de favorecer el crecimiento, las NIIF fortalecen los mercados e incrementan la inversión extranjera, algo en lo que coinciden Alsaqqa y Sawan (2013). En adición, la contabilidad internacional contribuye a la reducción de las desigualdades y la exclusión, debido a que los informes financieros emitidos bajo estándares internacionales son más transparentes para evaluar la rentabilidad potencial de las empresas, generando una promoción de la inversión que trae consigo un fortalecimiento de la economía nacional (Zaidi y Paz, 2015; Mhedhbi y Zeghal, 2016).

En palabras de Halbouni (2005) y Zaman Mir y Shiraz Rahaman (2005), las NIIF facilitan la toma de decisiones por parte de los grupos de interés. Además, estas disminuyen el riesgo sistémico y aumentan las posibilidades de rentabilidad de la inversión (Halbouni, 2005), haciendo que crezca el mercado de capitales (Rueda, 2010) y mejore la eficiencia de dicho mercado (Begoña, 2014).

Las firmas internacionales de auditoría se han beneficiado de todo este escenario (Halbouni, 2005). La consolidación y preparación de Estados Financieros, al igual que la auditoría, se facilitan en aras de la comparabilidad (Ufuk, Tucker y Yüikseltürk, 2013), para así “(...) evaluar y comparar el desempeño y las perspectivas de empresas de diferentes países" (Halbouni, 2005, p. 75).

Trabajos aplicados como el de Alemany i Costa (2007), al observar si la información contable publicada bajo NIIF para empresas españolas (variables básicas como el resultado neto y el valor neto patrimonial) influye en el 
Ruiz, L., Camargo, D. y Cardona O. Cambios en el valor bursátil de empresas colombianas listadas...

mercado de capitales, encuentran que los inversores tienen en cuenta otro tipo de datos diferentes a los contables. Para Europa, Armstrong, Barth, Jagolinzer y Riedl (2010), concluyeron que las expectativas del mercado respecto a los beneficios de la convergencia a NIIF fueron positivas, por las mejoras en la calidad de la información contable, que se hizo más simétrica entre agentes.

Ben Othman y Kossentini (2016) concluyen que para economías emergentes la adopción de las NIIF por parte de las empresas listadas afecta positivamente el mercado de capitales, y viceversa, porque como lo exponen Zeghal y Mhedhbi (2006) y Perera y Baudoun (2007), las NIIF no pueden funcionar satisfactoriamente en economías emergentes que no tienen mercados de capitales bien establecidos. A manera de ejemplo, Espinosa, Maquieira, Diaz y Abarca (2015), identificaron para Chile que la adopción de las NIIF no incidió en el precio de las acciones de las empresas listadas.

En Colombia, el informe ROSC (Reports on the Observance of Standards and Codes), elaborado por el Banco Mundial (2003), planteó que existían deficiencias manifiestas en la contabilidad local, debido al uso de los Principios de Contabilidad Generalmente Aceptados (PCGA), que consideraron como obsoletos e indescifrables para los inversionistas, además de reconocerlos como de baja calidad y que incluían principios de contabilidad en desuso. Por tal motivo, el Gobierno Nacional definió como uno de sus objetivos la necesidad de aplicar en las empresas los estándares propuestos por la contabilidad a nivel internacional, es decir, las NIIF. Para tal fin, en el país se empezó a hablar de la aplicación de las NIIF plenas en empresas listadas y no listadas en la BVC (Bolsa de Valores de Colombia, 2015).

Esta necesidad de Colombia, al igual que en los demás países, surgió de la necesidad manifiesta por adaptarse a las exigencias y acoger la contabilidad internacional, lo cual se deriva del proceso de globalización económica, para generar información contable armonizada desde las empresas, que permitiera la presentación de Estados Financieros comparables a nivel internacional (Bohórquez, 2015).

En efecto, mediante el proyecto de ley 203 de 2008, que posteriormente se convertiría en la Ley 1314 de 2009, el Congreso de la República de Colombia expidió el régimen normativo para los preparadores de información financiera. En esta ley se dio inicio al proceso de convergencia de las normas de contabilidad, de información financiera, y de aseguramiento de la información, con estándares internacionales aceptados a nivel mundial. Asimismo, se señalaron las autoridades competentes en materia contable, el procedimiento y las entidades responsables para vigilar su cumplimiento. De los decretos reglamentarios se tienen en el país las NIIF.

En el contexto colombiano, las investigaciones más recientes sobre NIIF se han orientado hacia la adopción de estas por parte de las pymes (Ruano, 
Vargas y Lasso, 2018; Ruiz y Perilla, 2019) y las mipymes (Gómez, 2017). En cuanto a trabajos empíricos, están los de Bedoya y Giraldo (2018) y Ruiz, Camargo y Cardona (2019). El primero de estos, muestra mediante el análisis de estadísticos descriptivos, que la adopción de las NIIF aumentó la liquidez del mercado para 23 empresas listadas en la BVC, así como su valor y rentabilidad del dividendo por acción. El segundo trabajo, por su parte, evaluó el efecto de las NIIF sobre los indicadores financieros ROE, OPM y ROIC (para 52 empresas listadas) y sobre el valor bursátil (para 32 empresas listadas), mostrando que no hubo cambios significativos en estas variables al darse el cambio normativo.

Para economías desarrolladas, el estudio de Lueg, Pawel y Burkett (2014), muestra que para empresas del Reino Unido, las variables margen operacional, retorno sobre el capital, retorno sobre el capital invertido, razón corriente y relación precio/ganancia se incrementaron después del uso de NIIF, lo que modificó las expectativas de los inversionistas en el mercado bursátil.

En Nueva Zelanda, Warwick, Bradbury y Hooks (2010) concluyen que para las compañías listadas en la bolsa de valores de Nueva Zelanda (NZX), la adopción de NIIF afectó las decisiones de valoración y crédito de las empresas por los cambios sucedidos en los indicadores financieros.

Para Finlandia, Lantto y Sahlström (2009), al investigar el efecto de la adopción de las NIIF en 91 empresas listadas en el mercado de valores de Helsinki, mostraron diferencias entre los indicadores financieros calculados bajo Finnish Accounting Standards (FAS) y bajo NIIF; por ejemplo, los de rentabilidad se aumentaron, dejando entrever cambios en las decisiones de inversión.

En Italia, Cordazzo (2013) muestra que para empresas listadas en bolsa el cambio hacia NIIF produjo un efecto positivo sobre los ingresos netos y el patrimonio, el cual fue más marcado en esta última variable. En esta misma línea de trabajo, Neag (2014) señala que para 67 empresas listadas en Rumania, el impacto de la aplicación de las NIIF en el ingreso neto y el patrimonio es relativamente más reducido. En las mediciones, estas dos variables fueron más bajas usando NIIF que bajo la regulación contable rumana (RAR por sus siglas en inglés).

\section{Metodología}

Para examinar el efecto que tuvo la convergencia a NIIF en el valor bursátil de empresas listadas en la $\mathrm{BCV}$, se hizo un análisis econométrico de regresión lineal usando como referencia el proceso metodológico seguido por García y Moya (2009), en donde no se consideraron variables del entorno macroeconómico, ni sectoriales. Se tuvieron en cuenta variables endógenas de las empresas en lo relacionado con su estructura económica, financiera y 
Ruiz, L., Camargo, D. y Cardona O. Cambios en el valor bursátil de empresas colombianas listadas...

de resultados del ejercicio, además de una variable de carácter exógeno que cuantificó el valor de las acciones de cada empresa.

La información se obtuvo de la base de datos de EMIS para 50 empresas pertenecientes a 5 de los 16 sectores establecidos por la BVC según se muestran en la Tabla 1. Estos datos correspondieron al valor de las acciones durante los años 2013, 2014, 2015 y 2016, y a los Estados Financieros en este mismo lapso de tiempo. Las empresas de la muestra se seleccionaron de 61 que tenían informes para el periodo descrito.

Tabla 1: Sectores de las empresas analizadas

\begin{tabular}{lc}
\hline \multicolumn{1}{c}{ Sector } & Cantidad \\
\hline Industrial & 34 \\
Inversión & 4 \\
Comercial & 1 \\
Servicios & 7 \\
Público & 4 \\
Total & $\mathbf{5 0}$ \\
\hline
\end{tabular}

Fuente: Elaboración propia.

Como los Estados Financieros obtenidos fueron para antes y después de la convergencia a NIIF, para los años 2013 y 2014, estos fueron elaborados por las empresas de acuerdo al Decreto 2649 de 1993 (PCGA); de otro lado, para los años 2015 y 2016, realizados con base en la Ley 1314 de 2009 y los decretos reglamentarios de las NIIF.

La variable dependiente correspondió al valor bursátil (VB) de las acciones de quienes pertenecen al Grupo 1, de acuerdo con la clasificación presentada en el Decreto 2784 de 2012 denominado "Marco Técnico Normativo para los Preparadores de Información Financiera que conforman el Grupo 1". Por su parte, las variables independientes empleadas se relacionan en la Tabla 2.

Tabla 2: Lista de variable independientes o explicativas

\begin{tabular}{lcc}
\hline \multicolumn{1}{c}{ Indicadores } & $\begin{array}{c}\text { Cuentas del estado de } \\
\text { situación financiera }\end{array}$ & Cuentas de resultados \\
\hline $\begin{array}{l}\text { De liquidez (L) = Activo Corriente / Pasivo } \\
\text { Corriente }\end{array}$ & Activo corriente (AC) & Ingresos ordinarios (F) \\
$\begin{array}{l}\text { De endeudamiento (RATEND) }=\text { Pasivos } \\
\text { Totales / Activos Totales }\end{array}$ & Activo no corriente (ANC) & Resultado Operacional (BN) \\
$\begin{array}{l}\text { De solvencia (S) }=\text { Activos Totales / Pasivos } \\
\text { Totales }\end{array}$ & Activo total (AT) & Resultado del ejercicio (BDI)
\end{tabular}




\begin{tabular}{lcc}
\hline \multicolumn{1}{c}{ Indicadores } & $\begin{array}{c}\text { Cuentas del estado de } \\
\text { situación financiera }\end{array}$ & Cuentas de resultados \\
\hline $\begin{array}{l}\text { Margen Bruto de Utilidad (MEX) = Utilidad } \\
\text { bruta / Ventas Netas }\end{array}$ & Pasivo corriente (ECP) \\
$\begin{array}{l}\text { Margen operacional (MO) }=\text { Utilidad } \\
\text { operacional / Ventas Netas }\end{array}$ & Pasivo no corriente (ELP) \\
$\begin{array}{l}\text { Rentabilidad de la situación líquida (RL) }= \\
\text { Utilidad operacional / Patrimonio } \\
\text { Rentabilidad del activo líquido (RAL) = Utilidad } \\
\text { Operacional/Disponible + Inversiones }\end{array}$ & Patrimonio neto (PN) \\
Rentabilidad económica (RE) = Utilidad & \\
Operacional + Gastos por intereses / Activos & \\
Totales & \\
Flujo de efectivo (CF)
\end{tabular}

Fuente: Elaboración propia a partir de García y Moya (2009).

Según lo anterior, la forma general del modelo para cada año y las empresas es:

$$
\begin{gathered}
V B_{i}=\beta_{\mathrm{o}}+\beta_{1} \mathrm{~L}_{i}+\beta_{2} \mathrm{RATEND}_{i}+\beta_{-} 3 \mathrm{~S}_{i}+\beta_{4} \mathrm{MEX}_{i}+\beta_{5} \mathrm{MO}_{i}+\beta_{6} \mathrm{RL}_{i}+ \\
\beta_{7} \mathrm{RAL}_{i}+\beta_{8} \mathrm{RE}_{i}+\beta_{9} \mathrm{CF}_{i}+\beta_{10} \mathrm{AC}_{i}+\beta_{11} \mathrm{ANC}_{i}+\beta_{12} \mathrm{AT}_{i}+\beta_{13} \mathrm{ECP}_{i}+ \\
\beta_{14} \mathrm{ELP}_{i}+\beta_{15} \mathrm{ET}_{i}+\beta_{16} \mathrm{PN}_{i}+\beta_{17} \mathrm{~F}_{i}+\beta_{18} \mathrm{BN}_{i}+\beta_{19} \mathrm{BDI}_{i}+\varepsilon_{i}
\end{gathered}
$$

Donde:

$\beta_{\mathrm{o}}=$ Intercepto.

$\beta_{1}^{\mathrm{o}}, \beta_{2}, \beta_{3}, \ldots, \beta_{19}=$ Estimadores.

$\varepsilon=$ Término de perturbación o error.

\section{Resultados}

Antes de iniciar con el análisis de regresión lineal, se presentan las correlaciones entre las variables independientes (antes y después de NIIF) y el VB (Tabla 3).

Tabla 3: Correlaciones entre variables independientes y dependiente

\begin{tabular}{cccccc}
\hline Tipo de Variable & $\begin{array}{c}\text { Variables } \\
\text { independientes }\end{array}$ & VB (2013) & VB (2014) & VB (2015 NIIF) & VB (2016 NIIF) \\
\hline AC & -0.0276 & 0.2047 & $0.3587^{*}$ & -0.0575 \\
& ANC & 0.1024 & $0.5493^{*}$ & $0.3669^{*}$ & $0.4062^{*}$ \\
Estado de Situación & AT & 0.2587 & 0.0625 & -0.0498 & -0.0368 \\
Financiera & ECP & -0.0374 & 0.1354 & -0.1639 & -0.1702 \\
& ELP & -0.0688 & $0.5119^{*}$ & 0.3289 & $0.3455^{*}$ \\
& ET & 0.3062 & $0.5139^{*}$ & 0.0453 & 0.1738 \\
& PN & 0.0987 & 0.1084 & 0.0931 & 0.1159 \\
\hline
\end{tabular}


Ruiz, L., Camargo, D. y Cardona O. Cambios en el valor bursátil de empresas colombianas listadas...

\begin{tabular}{cccccc}
\hline \multirow{2}{*}{ Tipo de Variable } & $\begin{array}{c}\text { Variables } \\
\text { independientes }\end{array}$ & VB (2013) & VB (2014) & VB (2015 NIIF) & VB (2016 NIIF) \\
\hline \multirow{3}{*}{ Estado de Resultados } & BDI & 0.0095 & $0.3299^{*}$ & 0.238 & 0.2464 \\
& BN & 0.0687 & 0.0425 & 0.2244 & 0.1329 \\
& F & -0.0231 & $0.3578^{*}$ & 0.2534 & 0.2624 \\
\hline Indicadores de & MEX & 0.0313 & 0.1642 & 0.2302 & 0.1253 \\
Rentabilidad & MO & $0.6840^{*}$ & $0.4032^{*}$ & 0.179 & 0.059 \\
& RAL & 0.1177 & 0.1439 & 0.0466 & 0.2883 \\
& RE & 0.2868 & $0.4054^{*}$ & $0.3595^{*}$ & $0.3656^{*}$ \\
Indicadores de liquidez, & RL & $0.3386^{*}$ & $0.5040^{*}$ & $0.4146^{*}$ & $0.3635^{*}$ \\
solvencia y endeudamiento & $\mathrm{CF}$ & 0.0646 & 0.1477 & 0.1207 & 0.067 \\
& $\mathrm{~L}$ & 0.1994 & 0.032 & 0.0526 & 0.0554 \\
& $\mathrm{~S}$ & 0.1404 & 0.1477 & -0.1649 & -0.1695 \\
\hline
\end{tabular}

Fuente: Elaboración propia.

Contrario a lo que podría pensarse, la Tabla 3 muestra a priori que los balances y razones financieras no están fuertemente correlacionadas con el VB. Además, de su falta de correlación, en general tampoco tienen una relación significativa al $5 \%$, independientemente del año y la norma contable que se haya usado para producir los datos.

La única variable significativa en los cuatro escenarios de VB fue el indicador de rentabilidad $\mathrm{RL}$, que a pesar de no relacionarse en un alto porcentaje (apenas entre un 33 a 50\%), su estadístico también resultó significativo en las regresiones ulteriores de esta sección.

Para calcular cada una de las regresiones se utilizaron tan solo las variables que se correlacionaron de forma estadísticamente significativa con la variable VB, según lo mostró la Tabla 3; paso seguido se eliminaron las variables que no cumplían con la prueba de significancia para el modelo lineal; de esta forma los modelos estimados se redujeron de 1 a 3 variables explicativas únicamente como se observa en las Tablas 4, 5, 6 y 7.

Tabla 4: Resultados de la regresión para el año 2013a,b

\begin{tabular}{cccccccc}
\hline \multirow{2}{*}{ Modelo } & \multicolumn{2}{c}{ Coeficientes no estandarizados } & Coeficientes estandarizados & \multirow{2}{*}{ t } & Sig. \\
\cline { 3 - 5 } \cline { 3 - 5 } 1 & B & Error estándar & Beta & & \\
\hline \multirow{2}{*}{1} & (Constante) & 8464,993 & 3859,354 & & 2,193 &, 035 \\
& RL & 74381,098 & 35447,514 &, 339 & 2,098 &, 043 \\
\hline
\end{tabular}

Nota: Variable dependiente: $\mathrm{VB} ; \mathrm{N}=36$ observaciones.

Fuente: Elaboración propia. 
Tabla 5: Resultados de la regresión para el año 2014,

\begin{tabular}{ccccccc}
\hline \multirow{2}{*}{ Modelo } & \multicolumn{2}{c}{ Coeficientes no estandarizados } & Coeficientes estandarizados & \multirow{2}{*}{ T } & \multirow{2}{*}{ Sig. } \\
\cline { 2 - 4 } & B & Error estándar & Beta & & 1,214 &, 233 \\
\cline { 3 - 5 } 1 & (Constante) & 4919,723 & 4051,591 & & 3,403 &, 002 \\
\hline & RL & 128139,736 & 37656,952 &, 504 & &
\end{tabular}

Nota: Variable dependiente: VB; $\mathrm{N}=36$ observaciones.

Fuente: Elaboración propia.

Las Tablas 4 y 5 contienen los resultados de las regresiones para los años 2013 y 2014 y que corresponden al periodo de tiempo analizado antes de que se aplicaran las NIIF. En ambos casos la rentabilidad como RL tuvo coeficientes de determinación $\mathrm{R}^{2}$ de 0.11 y 0.25 , respectivamente, lo cual no explicó sino un bajo porcentaje de la variación del VB.

Como resultado no fue posible afirmar que para los años inmediatamente anteriores a las NIIF, exista una relación estrecha entre las razones financieras, y muchos menos de los resultados contables con la valoración bursátil de las empresas.

Ahora bien, aplicando los mismos modelos de regresión para 2015 y 2016 (Tablas 6 y 7), se encontró que las variables significativas en el primero de estos años luego de la aplicación de las NIIF, fueron el AC (Activo corriente) y la RE (Rentabilidad económica).

Por otro lado, para el año 2016 las variables explicativas con significancia estadística menor al 5\% fueron el ANC (Activo no corriente), el ELP (Pasivo no corriente) y la RL (Rentabilidad de la situación líquida).

Tabla 6: Resultados de la regresión para el año 2015ª,b

\begin{tabular}{ccccccc}
\hline \multirow{2}{*}{ Modelo } & \multicolumn{2}{c}{ Coeficientes no estandarizados } & Coeficientes estandarizados & \multirow{2}{*}{ T } & \multirow{2}{*}{ Sig. } \\
\cline { 2 - 4 } & B & Error estándar & Beta & & \\
\hline \multirow{2}{*}{1} & (Constante) & $-1236,076$ & 5070,092 & &,- 244 &, 809 \\
& AC &, 0002873 &, 0000913 &, 455 & 3,145 &, 004 \\
& RE & 232239,669 & 60965,749 &, 551 & 3,809 &, 001 \\
\hline
\end{tabular}

Nota: Variable dependiente: VB; $\mathrm{N}=31$ observaciones.

Fuente: Elaboración propia.

Tabla 7: Resultados de la regresión para el año 2016 $6^{\mathrm{a}, \mathrm{b}}$

\begin{tabular}{|c|c|c|c|c|c|c|}
\hline & \multirow{2}{*}{ Modelo } & \multicolumn{2}{|c|}{ Coeficientes no estandarizados } & \multirow{2}{*}{$\begin{array}{c}\text { Coeficientes estandarizados } \\
\text { Beta }\end{array}$} & \multirow{2}{*}{$\mathrm{t}$} & \multirow{2}{*}{ Sig. } \\
\hline & & B & Error estándar & & & \\
\hline \multirow{4}{*}{1} & (Constante) & 4693,105 & 4415,572 & & 1,063 & ,296 \\
\hline & ANC &,- 0006244 & 0002821 &,- 479 & $-2,214$ &, 034 \\
\hline & ELP & ,0009101 & ,0003792 &, 516 & 2,400 & 022 \\
\hline & RL & 57666,469 & 16003,203 &, 532 & 3,603 & 001 \\
\hline
\end{tabular}

Nota: Variable dependiente: $\mathrm{VB} ; \mathrm{N}=37$ observaciones.

Fuente: Elaboración propia. 
Ruiz, L., Camargo, D. y Cardona O. Cambios en el valor bursátil de empresas colombianas listadas...

Dentro de los resultados para destacar de las Tablas 6 y 7 , se encuentra que las regresiones con la información elaborada bajo NIIF se asemejan a lo trabajado por García y Moya (2009), al cumplirse que las variables explicativas son representación del tamaño de la empresa y de la rentabilidad de estas, lo que no ocurrió con los análisis realizados con la información antes de NIIF.

Sumado a lo anterior, la estimación para el año 2015 explica un poco mejor la situación bursátil de las empresas con un coeficiente de determinación de 0.43 . Lo mismo sucede para el año 2016 del presente análisis, aunque el $\mathrm{R}^{2}$ continúa siendo muy bajo, alcanzando apenas el nivel de 0.32 .

La justificación más importante dentro de esos resultados es que las NIIF no repercutieron de forma considerable sobre la valoración que el mercado da a las acciones de las empresas negociadas en la BVC. Una explicación para estas estimaciones puede encontrarse en la disparidad de la información que existe, pues algunas empresas a pesar de tener versatilidad no cuentan con datos en bolsa de valores que se actualicen constantemente; esto pasa porque la mayoría de las negociaciones se da con las acciones de las compañías más representativas del país, es decir, que tienen mayor bursatilidad, que para el caso de la muestra analizada, no alcanzan la mitad para ninguno de los cuatro años.

\section{Conclusiones}

Acorde a los resultados del análisis de regresión lineal, las NIIF no afectaron considerablemente el grado de explicación de la valoración bursátil; esto significa que la contabilidad de las empresas en Colombia no tiene un grado de explicación considerable sobre el precio de las acciones, algo que sugiere que las cotizaciones responden a factores externos. Además, las empresas cotizantes en el país son pocas y la mayoría de las inversiones se concentran solo en un puñado de compañías.

En contradicción a lo planteado por Rueda (2010) y Alsaqqa y Sawan (2013), quienes consideran que las NIIF incrementan la inversión extranjera por mejoras en la bursatilidad, se observa en los hallazgos de este documento que en Colombia en el periodo en el que sucedió la convergencia no existieron variaciones significativas en el valor bursátil, situación similar a la sucedida en Chile (Espinosa, Maquieira, Díaz y Abarca, 2015).

Todo lo anterior es consistente con lo planteado por Ball (2016), quien afirma que los efectos de la adopción de las NIIF no necesariamente son visibles, lo que los hace difícilmente perceptibles.

De otro lado, se encontró que la variable rentabilidad líquida incidió sobre el valor bursátil para los años 2013, 2014 y 2016, lo que está alineado con el caso de Portugal, para el cual Miralles y Miralles (2002) muestran que el valor bursátil está influido por esta variable, además de otras como el activo neto, el flujo de efectivo, las deudas a terceros y las ventas. 
Para terminar, los resultados de este estudio a pesar de las limitaciones de los datos, podrían ser de cierto interés para examinar el alcance del impacto de las NIIF en el desempeño informado de las compañías listadas, por las comparaciones antes y después de la aplicación de los estándares contables.

\section{Referencias}

Alemany i Costa, J. (2007). La relevancia del valor de los datos contables en el mercado de valores español. Un estudio empírico para el período 1986-2003. Revista Española de Financiación y Contabilidad, 36(133), 199-202.

Alsaqqa, I. y Sawan, N. (2013). The Advantages and the Challenges of Adopting IFRS into UAE Stock Market. International Journal of Business and Management, 8(19), 1-23. http:// dx.doi.org/10.5539/ijbm.v8n19p1

Armstrong, C., Barth, M., Jagolinzer, A. y Riedl, E. (2010). Market Reaction to the Adoption of IFRS in Europe. The Accounting Review, 85(1), 31-61.

Ball, R. (2006). International financial reporting standards (IFRS): pros and cons for investors. Accounting and Business Research, 36(1), 5-27.

Ball, R. (2016). Why we do international accounting research. Journal of international accounting research, 15, 1-6. DOI: http://dx.doi.org/10.2139/ssrn.2724028

Banco Mundial. (2003). Colombia - Report on the observance of standards and codes (ROSC): accounting and auditing. $\mathrm{N}^{\circ}$ 35020. Obtenido el 15 de febrero de 2019 de: http:// documentos.bancomundial.org/curated/es/353391468241455747/pdf/350200CO0Ac counting0rosc 1 aa 1 col.pdf

Banco Mundial. (2005). Informe sobre el desarrollo mundial. Un mejor clima para la inversión en beneficio de todos. Obtenido el 15 de febrero de 2019 de: http://siteresources. worldbank.org/INTWDR2005/Resources/wdr2005_overview_spanish.pdf

Barth, M., Landsman, W., Lang, M. y Williams, C. (2012). Are IFRS-based and US GAAPbased accounting amounts comparable? Journal of Accounting and Economics, 54(1), 68-93.

Bedoya, B. y Giraldo, C. (2018). Adopción de las NIIF y su incidencia en la liquidez del mercado accionario de Colombia. Escenarios: empresa y territorio, 7(10), 1-19.

Begoña, G. (2014). Instituciones e intereses en conflicto ante la regulación contable internacional: el caso del sector financiero español. Revista de Contabilidad, 17(2), 143152. DOI: https://doi.org/10.1016/j.rcsar.2013.11.001

Baker, C. y Barbu, E. (2007). Trends in research on international accounting harmonization. The International Journal of Accounting, 42(3), 272-304. DOI: https://oi.org/10.1016/j. intacc.2007.06.003

Bohórquez, N. (2015). Implementación de norma internacional de inventarios en Colombia. Innovar, 25(57), 79-92.

Bolsa de Valores de Colombia. (2015). Adopción de Normas Internacionales de Información Financiera (NIIF o IFRS) en Colombia. Obtenido el 15 de febrero de 2019 de: https:// www.bvc.com.co/pps/tibco/portalbvc/Home/Empresas/NIIF?action=dummy 
Ruiz, L., Camargo, D. y Cardona O. Cambios en el valor bursátil de empresas colombianas listadas...

Botzem, S. (2014). Transnational standard setting in accounting: Organizing expertisebased selfregulation in times of crises. Accounting, Auditing \& Accountability Journal, 27(6), 933-955. DOI: https://doi.org/10.1108/AAAJ-04-2013-1301

Callao, S. y Jarne, J. (1995). La información Financiera en el contexto internacional. Revista española de financiación y contabilidad, 24(85), 937-968.

Carnegie, G. y Napier, C. (2002). Exploring comparative international accounting history. Accounting, Auditing \& Accountability Journal, 15(5), 689-718. DOI: https://doi. org/10.1108/09513570210448966

Católico, D. Urbina, J. y Gutiérrez, J. (2019). La incidencia de las Normas Internacionales de la Información Financiera sobre la revelación de la información contable en empresas colombianas. Apuntes Contables, (23), 85-105. https://doi.org/10.18601/16577175. n23.06

Cordazzo, M. (2013). The impact of IFRS on net income and equity: evidence from Italian listed companies. Journal of Applied Accounting Research, 14(1), 54-73. DOI: https://doi. org/10.1108/09675421311282540

Chandler, J. y Holzer, H. P. (1984). Accounting in the Third World. International Accounting. New York: Harper and Row.

Decreto 2649. Diario oficial No 41156, diciembre de 1993, Colombia.

Decreto 2784. Diario oficial No 48658, diciembre de 2012, Colombia.

De George, E., Li, X. y Shivakumar, L. (2016). A review of the IFRS adoption literature. Review of Accounting Studies, 21(3), 898-1004. DOI: https://doi.org/10.1007/s11142016-9363-1

El-Sayed, I. (2016). International accounting standards and accounting quality in code-law countries The case of Egypt. Journal of Financial Regulation and Compliance, 24(1), 4159. DOI: https://doi.org/10.1108/JFRC-12-2011-0047

Espinosa, C., Maquieira, C., Díaz, F. y Abarca, A. (2015). Adoption of IFRS in an emerging market: the Chilean case. Academia Revista Latinoamericana de Administración, 28(4), 442-460. DOI: https://doi.org/10.1108/ARLA-11-2014-0191

García, F. y Moya, I. (2009). Efecto de las NIIF en el valor bursátil de las empresas españolas. Investigaciones Europeas de Dirección y Economía de la Empresa, 15(1), 61-79.

Gómez, M. (2004). Una evaluación del enfoque de las Normas Internacionales de Información Financiera (NIIF) desde la teoría de la contabilidad y el control. Innovar, $24,112-131$.

Gómez, M. (2017). NIIF y MIPYMES: retos de la contabilidad para el contexto y la productividad. Cuadernos de Administración, 29(53), 49-76. DOI: https://doi. org/10.11144/Javeriana.cao29-53.nmrc

Halbouni, S. (2005). An empirical investigation of the perceived suitability of International Accounting Standards for Jordan. Journal of Economic \& Administrative Science, 21(1), 73-102. DOI: https://doi.org/10.1108/10264116200500004

Hamidah, Triyuwono, I., Sukoharsonoc, E. y Djamhurid, A. (2015). The Hegemony of International Interests on IFRS Adoption in Indonesia: An Accounting Ecology Perspective. Procedia - Social and Behavioral Sciences, 211(25), 104 - 110. DOI: https:// doi.org/10.1016/j.sbspro.2015.11.016 
Houqe, N. (2018). A review of the current debate on the determinants and consequences of mandatory IFRS adoption. International Journal of Accounting $\&$ Information Management, 26(3), 413-442. DOI: https://doi.org/10.1108/JJIM-03-2017-0034

Jarne, J. (1997). Clasificación y evolución internacional de los sistemas contables. Madrid: AECA. Lantto, A.-M., \& Sahlström, P. (2009). Impact of International Financial Reporting Standard adoption on key financial ratios. Accounting $\&$ Finance, 49(2), 341-361. DOI: https://doi. org/10.1111/j.1467-629X.2008.00283.x

Lasmin, R. (2012). Culture and the globalization of the international financial reporting standards (IFRS) in developing countries. Journal of International Business Research, 11(2), 31-44.

Ley 1314. Diario oficial No. 47409 de 13 de julio de 2009, Colombia.

Lueg, R., Pawel, P. y Burkett, M. (2014). Does transition to IFRS substantially affect key financial ratios in shareholder-oriented common law regimes? Evidence from the UK. Advances in Accounting, incorporating Advances in International Accounting, (30), 241-250. DOI: https://doi.org/10.1016/j.adiac.2014.03.002

Mhedhbi, K. y Zeghal, D. (2016). Adoption of international accounting standards and performance of emerging capital markets. Review of accounting and finance, 15(2), 252272. DOI: https://doi.org/10.1108/RAF-08-2013-0099

Miralles-Marcelo, J. L. y Miralles-Quirós, J. L. (2002). Factores determinantes del valor bursátil de las empresas portuguesas (1991-1999). Nuevas propuestas metodológicas. Revista Española de Financiación y Contabilidad, 31(112), 495-528.

Neag, R. (2014). The effects of IFRS on net income and equity evidence from Romanian listed companies. Procedia Economics and Finance, 15, 1787-1790. DOI: https://doi. org/10.1016/S2212-5671(14)00860-0

Nobes, C. y Parker, R. (2006). Comparative International Accounting. London: Prentice Hall.

Ben Othman, H. y Kossentini, A. (2016). IFRS adoption strategies and theories of economic development: Effects on the development of emerging stock markets. Journal of Accounting in Emerging Economies, 5(1), 70-121. DOI: https://doi.org/10.1108/JAEE-022012-0006

Perera, H. y Baudoun, N. (2007). Convergence with international financial reporting standards: The case of Indonesia. Advances in Internacional Accounting, 20, 201-224. DOI: https://doi.org/10.1016/S0897-3660(07)20007-8

Rodríguez, P. (2003). La contabilidad en Chile: una dimensión nacional e internacional. Panorama socioeconómico. Tesis Doctoral, 1-61.

Ruano, C., Vargas, C. y Lasso, G. (2018). Convergencia contable de las PyME colombianas. Cuadernos De Contabilidad, 19(47), 1-23. DOI: https://doi.org/10.11144/Javeriana.cc1947.ccpc

Rueda, G. (2010). El papel de la contabilidad ante la actual realidad económica, social y política del país. Más allá de la convergencia de prácticas mundiales. Cuadernos de Contabilidad, 11(28), 149-169.

Rueda, G. (2013). Contabilidad para la internacionalización y la globalización: hegemonía y disidencias. Papel Político, 18(1), 285-319. 
Ruiz, L., Camargo, D. y Cardona O. Cambios en el valor bursátil de empresas colombianas listadas...

Ruiz, L., Camargo, D. y Cardona, O. (2019). Effect of convergence to International Financial Reporting Standards on Companies Listed in the Colombian Stock Exchange. $A D$ minister, 35, 77-92. DOI: https://doi.org/10.17230/Ad-minister.35.3

Ruiz, M. y Perilla, L. (2019). Relevancia de factores socioeconómicos en la convergencia a NIIF: Análisis en Pymes de dos sectores de Villavicencio. Entramado, 15(1), 98-115. DOI: https://doi.org/10.18041/1900-3803/entramado.1.5363

Ufuk, I., Tucker, J. y Yuikseltuirk, O. (2013). Does Mandatory Adoption of IFRS Guarantee Compliance? The International Journal of Accounting, 48(3), 327-363. https://doi. org/10.1016/j.intacc.2013.07.002

Warwick, S., Bradbury, M. y Hooks, J. (2010). IFRS in New Zealand: effects on financial statements and ratios. Pacific Accounting Review, 22(2), 92-107. DOI: https://doi. org/10.1108/01140581011074494

Weirich, T., Avery, C. y Anderson, H. (1971). International accounting: Varying definitions. International Journal of Accounting, 7(1), 79-88.

Yip, R. y Young, D. (2012). Does mandatory IFRS adoption improve information comparability? The Accounting Review, 87(5), 1767-1789. DOI: https://oi.org/10.2308/ accr-50192

Zaidi, S. y Paz, V. (2015). The impact of IFRS adoption: a literature review. The Journal of Theoretical Accounting Research, 10(2), 116-141.

Zaman Mir, M. y Shiraz Rahaman, A. (2005). The adoption of international accounting standards in Bangladesh: An exploration of rationale and process. Accounting, Auditing \& Accountability Journal, 18(6), 816-841. https://doi.org/10.1108/09513570510627720

Zeghal, D. y Mhedhbi, K. (2006). An analysis of the factors affecting the adoption of international accounting standards by developing countries. The International Journal of Accounting, 41(4), 373-386. DOI: https://doi.org/10.1016/j.intacc.2006.09.009 